\title{
Development and assessment of EPInfant: a rating scale of perceived exertion for the pediatric population
}

\author{
Iván Rodríguez, Kinesiologista,b,c, Daniel Zenteno, M.D. ${ }^{c}$, \\ Laura Cisternas, Psychologist ${ }^{d}$, Paula Rodríguez, Psychologist ${ }^{c}$, \\ Gonzalo Reyes, Kinesiologist ${ }^{a}$, and Karen Troncoso, Kinesiologist ${ }^{a}$
}

a. School of Kinesiology, School of Health Sciences, Universidad San Sebastián.

b. School of Kinesiology, School of Medicine, Universidad Santo Tomás.

c. Department of Pediatrics, Hospital Dr. Guillermo Grant Benavente.

d. Sagrado Corazón de Jesús School. Chile

E-mail Address:

Iván Rodríguez,

Kinesiologist: ivan. rodriguez@uss.cl

\section{Funding:}

The study was funded by the School of Kinesiology of Universidad San Sebastián. Chile.

Conflict of Interest: None.

Received: 11-9-2014 Accepted: 6-18-2015

\section{ABSTRACT}

Rating scales of perceived exertion(PE) have been used to measure PE during exercise in the adult population with successful results. However, in children, it is necessary to develop adapted instruments due to their cognitive immaturity. The number of Spanish-language validated instruments for the pediatric population is scarce. Our objective was to develop a new PE rating scale. Research design was divided into three stages: development, content validity, and criterion validity. For development, a panel of five expert members was summoned, who provided indications for the new instrument. A systematic review of the literature to identify other validated instruments was performed. This process resulted in the development of the new EPInfant scale to measure PE, which integrates the expert panel's indications and the result of the literature systematic review. The evaluation of its content showed a high level of agreement regarding the quality of its design; the content validity index was 1 . During the field assessment, a high concurrent criterion validity was observed in healthy adolescents due to the strong correlation shown between PE and heart rate in both boys and girls.

The methodology used to develop the EPInfant scale aimed at reducing potential biases that may hinder its psychometric properties. Preliminary results suggest that this may be a validated instrument that could be implemented in healthy adolescents.

Key words: child, adolescent, exercise, cognition, exertion, perception.

http:/ /dx.doi.org/10.5546/aap.2015.eng.550

\section{INTRODUCTION}

Perceived exertion (PE) rating scales are used to measure the feeling caused by metabolic changes during exercise..$^{1-3}$ These instruments were initially developed and validated for the adult population; the first version was developed by Borg. ${ }^{1}$ The Borg Scale is a numerical vertical scale with a rating of 6-20 and its objective is to provide perceptual data in correlation to heart rate (HR) and workload. The purpose of such numerical distribution is to establish an association between the level of PE (multiplied by 10) and HR during exercise. $^{1}$

PE measurement has been used in varying settings, both as a tool to measure physiological stress and to establish exercise intensity. ${ }^{4-6}$ According to Borg's model, as exercise intensity increases, PE increases accordingly in response to progressive activity intensities, which demonstrates a positive correlation between both outcome measures. ${ }^{1-8}$ However, since children's cognitive development is not sufficient to understand numerical descriptors of physiological stress used for adults, it is necessary to develop scales especially designed for the pediatric population with characteristics expressing levels of physiological stress during exercise in a particular manner. ${ }^{9-13}$ Several instruments have been developed that have shown an acceptable performance in children and adolescents of different age groups and cultures. ${ }^{10-18}$

However, the number of adequately-developed and validated scales in Spanish language is scarce. ${ }^{19}$ In Chile, several initiatives have resulted in the development of tools to measure PE in children, including the Pediatric Visual Analog Scale (PVAS), which is an adaptation of the Borg Scale for the pediatric population. ${ }^{20}$ To date, its psychometric properties have not been established and its clinical usefulness has shown inconsistent results. ${ }^{21}$ In addition, the PVAS is derived from the Borg Scale (adaptation), which has demonstrated a limited concurrent criterion validity 
in the pediatric population. ${ }^{18}$ Based on this, there is a need to introduce major modifications in the PVAS in order to generate an instrument that is adequately adapted for the pediatric population, in Spanish language and with methodological characteristics that allow its valid implementation in the target population.

This new measuring instrument was developed using a methodology aimed at reducing potential biases that may affect its psychometric properties. ${ }^{22}$ Research design was divided into three stages: development, content validity, and concurrent criterion validity (Figure 1).

Below we describe each stage in the preparation of this new perceived exertion rating scale for children (EPInfant).

\section{DEVELOPMENT OF THE EPInfant SCALE}

The EPInfant was developed based on the recommendations for the development of health measurement instruments ${ }^{22}$ which suggest considering the opinions of an expert panel as well as theoretical background obtained by means of a systematic review of the literature (SRL). In our case, we requested the opinion of an expert panel to establish the instrument's characteristics for an adequate PE rating on the basis of a consensus. Simultaneously, we performed an SRL to identify other scales that have been adequately validated for the pediatric population.
The expert panel was made up of five Chilean health care professionals with more than four years of experience and verifiable training in the fields of health and child education (two psychologists, two kinesiologists and a pediatrician specialized in bronchopulmonary conditions). As per the Delphi method, ${ }^{23}$ each expert separately received a manuscript containing general information on $\mathrm{PE}_{,}^{24}$ an image of the PVAS, ${ }^{25}$ and three open questions aimed at making possible changes to said instrument: ${ }^{1} \mathrm{Do}$ you think the PVAS has the necessary elements for 6-18 year-old children and adolescents to interpret it? Please indicate which elements. ${ }^{2}$ Do you think the PVAS should include other elements in order to improve its validity based on the type of thinking according to the child's psychological development? Please indicate which elements. ${ }^{3}$ Do you think the PVAS should suppress certain pictorial elements in order to improve its psychometric properties based on the child's cognitive development? Please indicate which elements.

To sum up, the five members of the expert panel agreed on the fact that, for question 1, the new instrument should maintain the purpose (of the PVAS) of representing the level of PE and physiological stress by means of pictures and numbers that would allow children to interpret it intuitively. For question 2, experts suggested the introduction of verbal descriptors of PE

FIgURE 1. Research design

Development

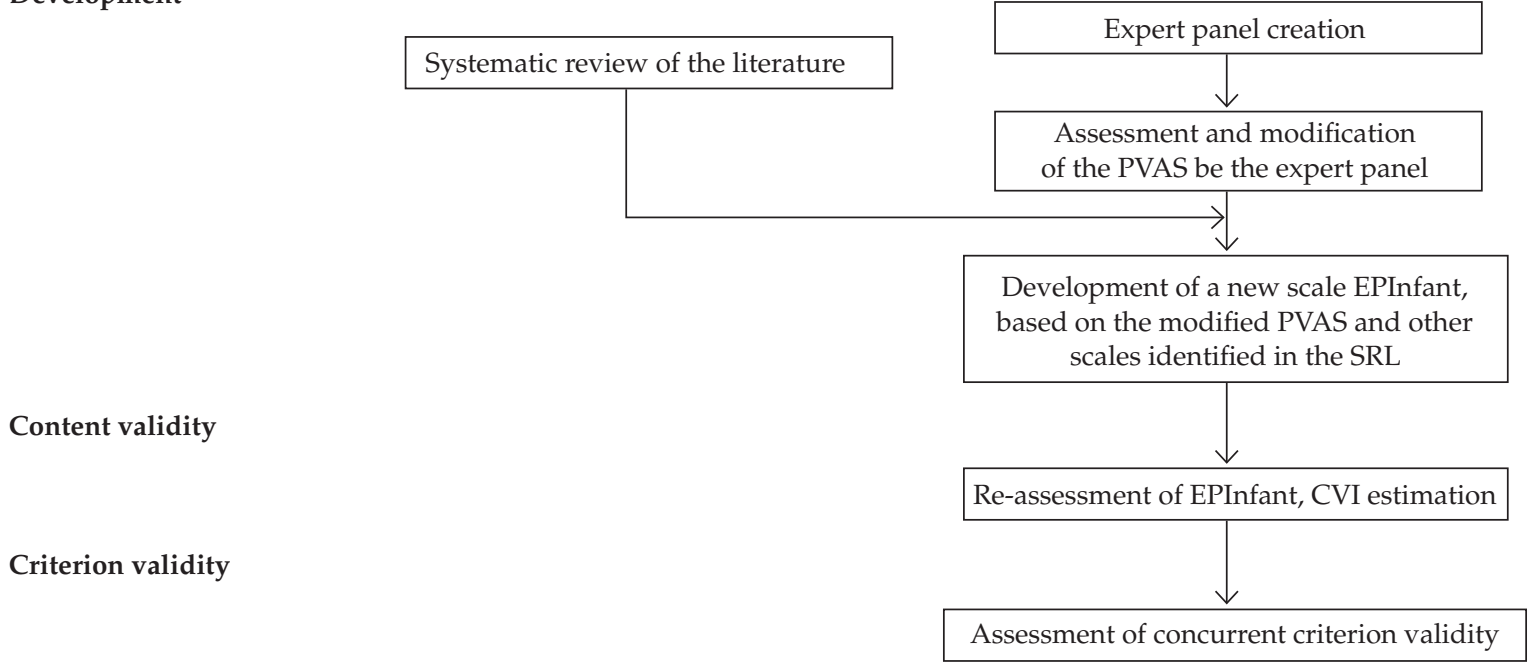

PVAS: Pediatric Visual AnalogScale; CVI: content validity index;

EPInfant: new pediatric perceived exertion rating scale; SRL: systematic review of the literature. 
in Spanish, using a clear language targeted at children, as well as pictures with better quality and resolution (compared to the PVAS). For this question, only one expert suggested that bar height should increase in an exponential leftto-right manner instead of in a linear manner, as depicted in the PVAS. Finally, for the last question, the entire panel suggested that the scale should not include images that represent other constructs (sun, moon, or mountains, which are included in the PVAS).

For its part, based on the SRL, studies conducted in childrenthat established the correlation between $\mathrm{PE}$ and a physiological outcome measure $\left(\mathrm{VO}_{2}\right.$ and $\mathrm{HR}$ ) during a standardized exercise test were selected ${ }^{26}$ For each primary article, Pearson's $r$ correlation coefficient (CC) was recorded, which was weighted according to the sample size to estimate a general weighted average. Only scales with a weighted CC higher than the general weighted average were considered. The following scales were selected: the perceived exertion scale for children, ${ }^{27}$ the cart and load effort rating, ${ }^{17}$ the Eston-Parfitt scale of perceived exertion, ${ }^{28,29}$ the pictorial version of the Children's Effort Rating Table, ${ }^{16,30}$ and the "OMNI" ratings of perceived exertion scale. ${ }^{15-18,31,32}$ Based on these five selected scales, pictorial, numerical and verbal descriptors were considered to develop the EPInfant (the details on the SRL methodology are shown in the supplementary annex in electronic format spanish version).

The EPInfant scale was designed by integrating experts' indications and methodological elements observed in the design of those scales with the highest CVI identified in the SRL (Figure 2). In addition, and during the development process, a set of recommendations for use was prepared in order to standardize instructions for an adequate instrument use (Table 1).

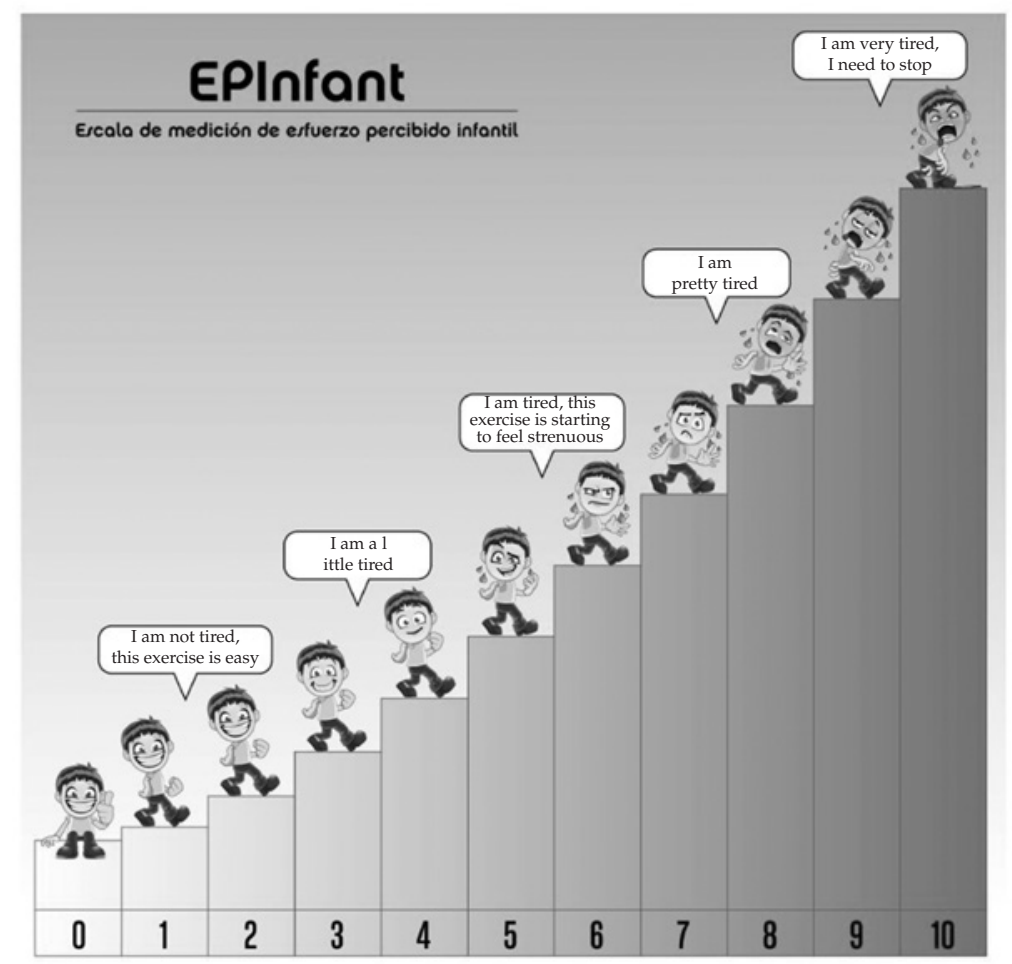

The scale shows 11 numerical descriptors (0 to 10), five verbal descriptors located each every two intensity levels, and a set of pictures that illustrate a child running at progressively increasing intensities across a scale made up of bars that increase in height with an exponential, left-to-right slope. 


\section{CONTENT VALIDITY}

The next stage was content validity, which accounts for the extent to which the scale measures the construct of interest. The measurability method here is the content validity index (CVI). This indicates the degree of agreement among a group of experts in relation to the relevance of items included in the new measurement instrument. ${ }^{33}$ Given that the EPInfant scale is not based on items, CVI was measured based on its quality. To this end, each member of the expert panel assessed the EPInfant scale by establishing a certain level of agreement with different statements on its methodological quality. Agreement levels were established based on literature recommendations, ${ }^{33}$ as follows: 0 : strongly disagree; 1 : disagree; 2 : agree; 3 : strongly agree. The proportion of answers in agreement with each statement was regarded as an index of agreement, and the proportion of answers with some level of agreement with each statement (2: agree; 3: strongly agree) was considered the CVI. As a result, the index of agreement among experts was 0.86 and CVI was 1 ; this demonstrates that the expert panel agreed that the methodological quality of the EPInfant scale was adequate for the construct (Table 2).

\section{CONCURRENT CRITERION VALIDITY}

For the last stage in this process, a study was conducted to assess the correlation between HR and PE (EPInfant) during the exercise test. To this end, healthy adolescents from a public school in Concepción, Chile, were selected. Subjects with cognitive impairment, obesity or motor neurone disease that prevented them from doing exercise were excluded.

Protocol: The exercise test used was the Chester test (CT), which consists in going up and down a $20 \mathrm{~cm}$ step at an increasing pace set by a signal sound. It includes five 2-minute levels; for each level, the number of cycles to be done in 1 minute by the patient increases $($ cycle $=$ each foot stepping up and then each foot stepping down): level 1: 15 cycles; level 2: 20 cycles; level 3: 25 cycles; level 4: 30 cycles; level 5: 35 cycles. During the last 20 seconds of each minute, PE, $\mathrm{HR}$ and oxygen saturation $\left(\mathrm{SatO}_{2}\right)$ are recorded. Theoretical maximum HR is estimated based on the following formula: 208 - (0.7 $x$ age).

HR was recorded using a $\mathrm{Polar}^{\circledR}$ cardiorespiratory monitor, $\mathrm{SatO}_{2}$ was recorded using a NONIN ${ }^{\circledR}$ pulse oximeter. Before doing the test, subjects became familiar with the EPInfant scale and had time to consider its recommendations for use.

Statistical analysis schedule: Assuming a type I error of $5 \%$, a type II error of $80 \%$ and a Pearson's $r$ coefficient of 0.7 , the necessary sample was 14 subjects per sex. Considering that the sample was made up of 40 adolescents (20 boys and 20 girls), the study's estimated statistical power was $95 \%$. Once the sample's normality was verified through the Shapiro-Wilk test, average

\section{TABLE 1. Recommendations for the implementation of the EPInfant pediatric perceived exertion rating scale}

- The EPInfant scale is an instrument designed to measure whole-body perceived exertion in children and adolescents (younger than 18 years old) during physical exercise.

- This scale should be introduced before performing physical exercise and explained in terms that are simple and appropriate for the subject's age.

- If the child cannot read, pictures of children doing exercise should be used to provide instructions aimed at interpreting perceived exertion.

- For an adequate measurement of perceived exertion, subjects should answer the question "How tired do you feel during exercise?". This question should aim at assessing whole-body perceived exertion, including weariness in the legs and dyspnea.

Below, there is an example on how to adequately provide instructions:

- Before, during and after exercise, I will ask you how tired you feel.

- You should use the numbers, words or pictures of children to indicate your level of tiredness during exercise.

- Please, watch the picture of the child at the beginning of the scale. If you feel like him, it means you are not tired.

- Please, watch the pictures of the children in the middle of the scale (levels 5 and 6). If you feel like any of them, it means you are tired, but you may continue doing exercise.

- Please, watch the picture of the child at the end of the scale. If you feel like him, it means you are very tired and cannot continue doing exercise.

- You may use any of the numbers, statements and/or pictures of children in the scale to describe how tired you feel. There are no right or wrong answers. 
and standard deviation values were estimated. An ANOVA for repeated measures was used to assess differences between HR and PE at the different exercise intensity levels; a t test for independent samples was used to compare PE and HR at the end of the test between boys and girls. In addition, criterion validity was assessed by estimating Pearson's r CC between average $\mathrm{HR}$ and PE values in boys and girls during the exercise test. A $p$ value $<0.05$ was considered significant. The MedCalc software, version 14.12.0 (MedCalc Software bvba, Ostend, Belgium) was used.

Assessment results: The average age of participants in the sample was $14.2 \pm 0.8$ years old, with a normal height and weight. The mean HR reached was $150.3 \pm 17.1$ (76\% of maximum HR) and $171.2 \pm 11.4(86 \%$ of maximum HR) in boys and girls $(p<0.0001)$. In addition, the level of PE reached was $2.8 \pm 1.4$ and $3.8 \pm 2.3$ in boys and girls, respectively. In turn, PE increased significantly with each increasing level of exercise intensity $(p<0.05)$ (Figure 3). Besides, a strong correlation between PE and HE was observed throughout the test in boys and girls $(r=0.93$, $p<0.0001$ and $\mathrm{r}=0.95, p<0.0001$, respectively). No significant difference was observed in the degree of correlation between PE and HR between both sexes (Figure 4).

\section{COMMENT}

$\mathrm{PE}$ is a subjective assessment of the intensity of exercise done, which is made up of a set of feelings that integrates stress and fatigue in the muscle-skeletal, cardiovascular and respiratory systems during exercise. ${ }^{8,24}$ This allows to adequately regulate physical fitness so that tasks may be completed without exceeding a physiological tolerance threshold. ${ }^{1,24}$

No data have been reported from a neurophysiological perspective that allow to verify whether cognitive function influences $\mathrm{PE}$; however, PE has been observed to increase based on children's cognitive development stages. ${ }^{8}$ For this reason, it has been suggested that scales be adapted to the pediatric population in order to obtain valid and reliable PE measurements. ${ }^{14}$

FIGURE 3. Perceived exertion as per progressive excercise intensity level

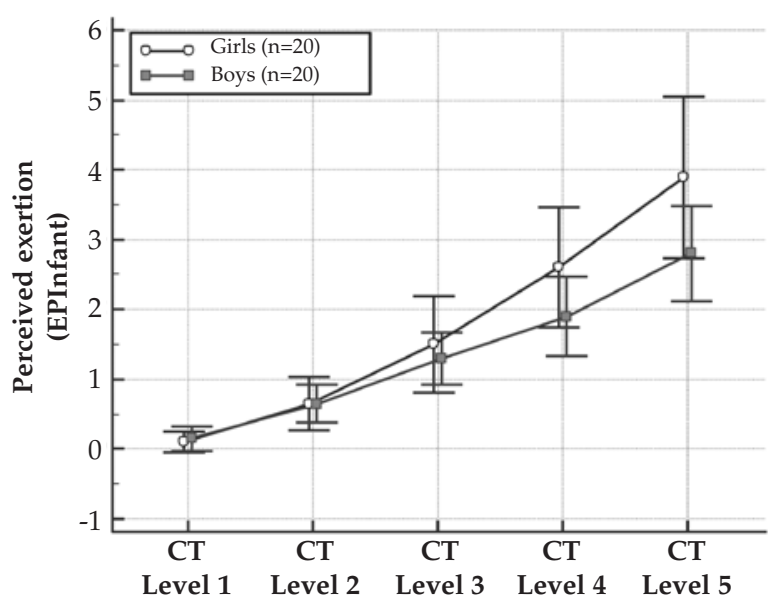

* CT Levels 1-5: these account for exercise intensity levels during the Chester test.

TABLE 2. Content assessment

\begin{tabular}{lcc}
\hline Statements & Concordance \\
\hline $\begin{array}{l}\text { The scale pictures have the necessary elements } \\
\text { for 6-12 year-old children to interpret it. }\end{array}$ & 0.8 \\
$\begin{array}{l}\text { The scale pictures have the necessary elements } \\
\text { for 13-15 year-old children to interpret it. }\end{array}$ & 1 \\
$\begin{array}{l}\text { Numerical and verbal descriptors are adequate } \\
\text { for 6-12 year-old children to interpret them. }\end{array}$ & 0.8 \\
$\begin{array}{l}\text { Numerical and verbal descriptors are adequate } \\
\text { for 13-15 year-old children to interpret them. }\end{array}$ & 1 \\
$\begin{array}{l}\text { Recommendations for use are adequate to provide instructions } \\
\text { on the instrument to 6-12 year-old children. }\end{array}$ & 0.6 \\
$\begin{array}{l}\text { Recommendations for use are adequate to provide instructions } \\
\text { on the instrument to 13-15 year-old children. }\end{array}$ & 1 \\
\begin{tabular}{l} 
General average \\
\hline
\end{tabular} & 1 \\
\hline
\end{tabular}

CVI (content validity index): proportion of answers with some level of agreement with each statement (2: agree; 3 : strongly agree). 
The EPInfant scale includes pictorial aids that may potentially favor its interpretation considering different cognitive development stages, ${ }^{8}$ such as pictures, colors, increasing height bars (preoperational stage [5-7 years old] and concrete operational stage [8-12 years old]), as well as numbers and verbal descriptors to describe exercise intensity (formal operational stage [13-18 years old]). This was supported by the expert panel, which showed a high level of agreement with the scale's quality.

Once developed and validated by the expert panel, any new scale should be assessed in different implementation settings. For this reason, first of all we considered a test with increasing exercise levels that was safe and easily performed, such as the step test, in order to focus on measuring PE instead of on the exercise test performance process. Research design considered the perceptual estimation paradigm, which uses PE to measure perceived intensity during an exercise test. CC observed between $\mathrm{HR}$ and $\mathrm{PE}$ was used as criterion validity index. Therefore, the CC observed both in boys and girls corresponded to $r=0.93$ and $r=0.95$, which suggests that the EPInfant scale may have an adequate performance during exercise in this group of subjects.

Similar results have been observed in other studies. Pfeiffer, et al. observed a strong correlation between PE and HR in adolescents during an increasing exercise test using a treadmill. ${ }^{18}$ On their side, Robertson, et al. observed correlation levels of 0.83 and 0.88 in both sexes during a step exercise test. ${ }^{31}$

FIGURE 4. Correlation between preceived exertion and heart rate

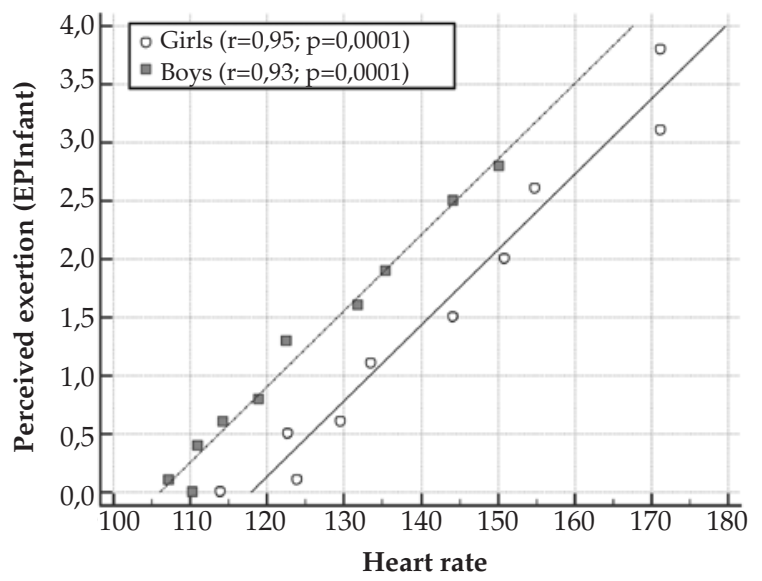

To date, no other field validity studies have been conducted that consider other age groups and other exercise types and modalities, so it is not possible to extrapolate these results to the overall pediatric population. However, these results are encouraging to continue studying the performance of the EPInfant scale based on its high content validity (agreement among experts) and an adequate criterion validity in adolescents, two characteristics that had been questioned and completely unknown with the instrument available to date.

Future studies should not only consider other age groups and clinical settings, but should also include both cognitive function assessment paradigms during exercise in their methodological designs, i. e., the estimation paradigm (the instrument's ability to measure PE) and the output paradigm (dosing of exercise intensity based on PE). In addition, it is necessary to assess the scale's reliability in order to know the expected degree of variability between repeated measures over time and among different observers. A thorough knowledge of these data will provide a solid support to validate the instrument in the general pediatric population and it will therefore allow to recommend it in different settings that are related to physical therapy and exercise practice in children and adolescents.

\section{REFERENCES}

1. Borg G. Perceived exertion as an indicator of somatic stress. Scand J Rehabil Med 1970;2(2): 92-8.

2. Smirmaul B de P. Sense of effort and other unpleasant sensations during exercise: clarifying concepts and mechanisms. Br J Sports Med 2012;46(5):308-11.

3. Marcora S. Perception of effort during exercise is independent of afferent feedback from skeletal muscles, heart, and lungs. J Appl Physiol (1985) 2009;106(6):2060-2.

4. Dunbar CC, Robertson RJ, Baun R, Blandin MF, et al. The validity of regulating exercise intensity by ratings of perceived exertion. Med Sci Sports Exerc 1992;24(1):94-9.

5. Buckley JP, Eston RG, Sim J. Ratings of perceived exertion in braille: validity and reliability in production mode. $\mathrm{Br}$ J Sports Med 2000;34(4):297-302.

6. Hartshorn JE, LambKL. The reproducibility of perceptually regulated exercise responses during short-term cycle ergometry. Int J Sports Med 2004;25(5):362-7.

7. KangJ,Chaloupka EC, Biren GB,MastrangeloMA,Hoffman JR. Regulating intensity using perceived exertion: effect of exercise duration. Eur J Appl Physiol 2009;105(3):445-51.

8. Groslambert A, Mahon AD. Perceived exertion: influence of age and cognitive development. Sports Med 2006;36 (11):911-28.

9. Williams JG, Eston R, Furlong B. CERT: a perceived exertion scale for young children. Percept Mot Skills 1994;79(3 Pt2):1451-8.

10. Eston RG, Lamb KL, Bain A, Williams AM, Williams 
JG.Validity of a perceived exertion scale for children: a pilot study. Percept Mot Skills 1994;78(2):691-7.

11. Robertson RJ, GossFL, BoerNF, Peoples JA, etal.Children's OMNI scale of perceived exertion: mixed gender and race validation. Med Sci Sports Exerc 2000;32(2):452-8.

12. Eston RG, Parfitt G, Campbell L, Lamb KL. Reliability $\mathrm{f}$ effort perception for regulating exercise intensity in children using the Cart and Load Effort Rating (CALER) Scale. Pediatr Exerc Sci 2000;12(4):388-97.

13. Yelling M, Lamb KL, Swaine I. Validity of a pictorial perceived exertion scale for effort estimation and effort production during stepping exercise in adolescentchildren. Eurphy Seduc Rev 2002;8:157-75.

14. Groslambert A, Hintzy F, Hoffman MD, Dugué B, Rouillon JD. Validation of a rating scale of perceived exertion in young children. Int J Sports Med 2001;22(2):116-9.

15. Utter AC, Robertson RJ, Nieman DC, Kang J. Children's OMNI Scale of Perceived Exertion: walking/running evaluation. Med Sci Sports Exerc 2002;34(1):139-44.

16. Roemmich JN, Barkley JE, Epstein LH, Lobarinas CL, et al. Validity of PCERT and OMNI walk/run ratings of perceived exertion. Med Sci Sports Exerc 2006;38(5):1014-9.

17. Barkley JE, Roemmich JN. Validity of the CALER and OMNI-bike ratings of perceived exertion. Med Sci Sports Exerc 2008;40(4):760-6.

18. Pfeiffer KA, Pivarnik JM, Womack CJ, Reeves MJ, Malina RM. Reliability and validity of the Borg and OMNI rating of perceived exertion scales in adolescent girls. Med Sci Sports Exerc 2002;34(12):2057-61.

19. Suminski RR, Robertson RJ, Goss FL, Olvera N. Validation of the Omni Scale of Perceived Exertion in a sample of Spanish-speaking youth from the USA. Percept Mot Skills 2008;107(1):181-8.

20. Zenteno D, Puppo H, Vera R, Torres R, et al. Guías de rehabilitación para niños con enfermedades respiratorias crónicas. Neumol Pediatr 2007; 3 (Suppl 1):25-33.

21. Rodríguez I, Henríquez S, Vásquez P, Zenteno D. Test de caminata de seis minutos y función pulmonar en pacientes con bronquiolitis obliterante post infecciosa. Rev Chil Enferm Respir 2014;30(2):68-74.

22. Benson J, Clark F. A guide for instrument development and validation. Am J OccupTher 1982;36(12):789-800.

23. Varela-RuizM,Díaz-BravoL,García-Durán R. Descripcióny usos del método Delphi en investigaciones del área de la salud. Inved Med 2012;1(2):90-5.

24. Borg GA. Psychophysical bases of perceived exertion. Med Sci Sports Exerc 1982;14(5):377-81.

25. Zenteno D, Puppo H, González R, Kogan R. Test de marcha de 6 minutos en pediatría. Neumol Pediatr 2007;2(2):109-14.

26. Chen MJ,FanX,MoeST.Criterion-related validity of the Borg ratings of perceived exertion scale in healthy individuals: a meta-analysis. J Sports Sci 2002;20(11):873-99.

27. Cassady SL, Kaufman BA, Kelly CE, Eisenmann SC, et al. Validity of a New Perceived Exertion Scale for Children. Cardio Pulmphys Ther J 1998;9(1):3-8.

28. Eston RG, Lambrick DM, Rowlands AV. The perceptual response to exercise of progressively increasing intensity in children aged 7-8 years: validation of a pictorial curvilinear ratings of perceived exertion scale. Psycho Physiology 2009;46(4):843-51.

29. Lambrick DM, Rowlands AV, Eston RG. The perceptual response to treadmill exercise using the Eston-Parfitt scale and marble dropping task, in children age 7 to 8 years. Pediatr Exerc Sci 2011;23(1):36-48.

30. Marinov B, MandadjievaS, Kostianev S. Pictorial and verbal category-ratio scales for effort estimation in children. Child Care Health Dev 2008;34(1):35-43.

31. Robertson RJ, Goss FL, Andreacci JL, Dubé JJ, et al. Validation of the children s OMNI RPE scale for stepping exercise. Med Sci Sports Exerc 2005;37(2):290-8.

32. Balasekaran G, Loh MK, Govindaswamy VV, Robertson RJ. OMNI Scale of Perceived Exertion: mixed gender and race validation for Singapore children during cycle exercise. Eur J Appl Physiol 2012;112(10):3533-46.

33. PolitDF, BeckCT,OwenSV.IstheCVIanacceptableindicator of content validity? Appraisal and recommendations. Res Nurs Health 2007;30(4):459-67. 